\title{
Perioperative considerations for neoadjuvant immunotherapy in non-small cell lung cancer
}

Brendon M. Stiles, MD, ${ }^{\mathrm{a}}$ Boris Sepesi, MD, ${ }^{\mathrm{b}}$ Stephen R. Broderick, MD,${ }^{\mathrm{c}}$ and Matthew J. Bott, $\mathrm{MD}^{\mathrm{d}}$

Feature Editor's Introduction-The mortality of lung cancer has declined over the past decade at record rates of up to $4.3 \%$ per year, ${ }^{1}$ and immune checkpoint inhibitors (ICIs) are at least partly responsible for this trend. On the basis of efficacy, ICIs have rapidly increased from investigational agents to standard of care for metastatic non-small cell lung cancer (NSCLC). Not long after demonstrating improved survival compared with standard chemotherapy in patients with stage IV disease, ICIs entered clinical trials for earlier stage lung cancer. For example, the programmed death ligand 1 inhibitor durvalumab has recently been adopted as standard of care for patients with locally advanced (stage IIIB) NSCLC after its addition to chemoradiation was shown to improve survival. These and other promising data in advanced NSCLC data have ushered ICIs into the neoadjuvant setting, and clinical trials for stage IB to IIIA NSCLC have completed or are accruing. On the basis of the early output of these trials (reviewed herein) and the track record of ICIs in advanced NSCLC, it is reasonable to suspect that there will be a place for ICIs in the standard of care for early-stage disease. This is a new frontier in thoracic surgery, and operating on patients who have received immunotherapy is becoming commonplace in many institutions. However, there are challenges with neoadjuvant ICI therapy that are not obvious and that are directly relevant to thoracic surgeons. Aside from operative considerations such as the hilar fibrosis that can result from ICIs, and choosing the optimal approach, there are new concepts such as nodal immune flare and pseudo-progression that must be understood and considered in restaging and surgical decision-making.

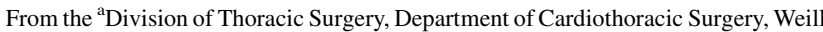
Cornell Medicine, New York-Presbyterian Hospital, New York, NY; ${ }^{\mathrm{b}}$ Department of Thoracic and Cardiovascular Surgery, The University of Texas MD Anderson Cancer Center, Houston, Tex; ${ }^{\circ}$ Division of Thoracic Surgery, Department of Surgery, The Johns Hopkins Medical Institutions, Baltimore, Md; and ${ }^{\mathrm{d}}$ Thoracic Service, Department of Surgery, Memorial Sloan Kettering Cancer Center, New York, NY.

Received for publication Feb 10, 2020; revisions received May 12, 2020; accepted for publication May 13, 2020; available ahead of print July 5, 2020.

Address for reprints: Brendon M. Stiles, MD, Division of Thoracic Surgery, Department of Cardiothoracic Surgery, Weill Cornell Medicine of Cornell, University, Suite M404, 525 East 68th St, New York, NY 10065 (E-mail: brs9035@med. cornell.edu).

J Thorac Cardiovasc Surg 2020;160:1376-82

$0022-5223 / \$ 36.00$

Copyright $(\odot 2020$ by The American Association for Thoracic Surgery https://doi.org/10.1016/j.jtcvs.2020.05.119

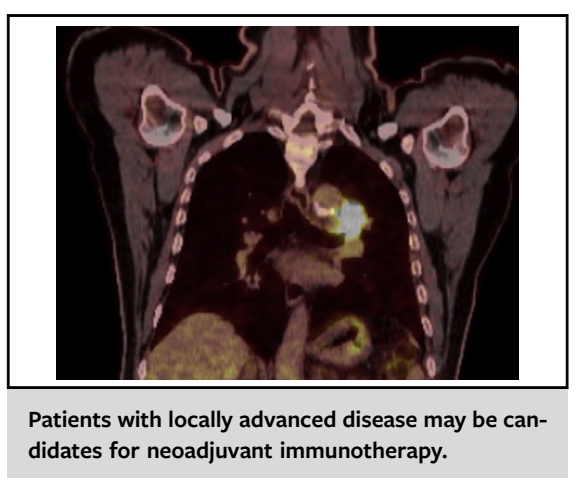

CENTRAL MESSAGE

Intense interest exists in preoperative immunotherapy for patients with surgically resectable lung cancer. Surgeons should be actively involved in trial design, patient selection, and outcome evaluation.

See Commentary on page 1383 .

There are new immune-related adverse events and complications germane to preoperative risk stratification and postoperative management (eg, pneumonitis). In the following Expert Opinion article, thoracic surgical oncologists at the forefront of this field offer a succinct and practical primer on preoperative, intraoperative, and postoperative considerations for neoadjuvant ICI therapy in NSCLC. Included also is an expert summary of published and presented neoadjuvant ICI efficacy data that should rally our discipline to pile on to the lung cancer mortality curve and lead its next record decline.

\section{Bryan, M. Burt, MD}

Given the success of immune checkpoint blockade (ICB) in patients with metastatic lung cancer, intense interest exists in moving these therapeutics into earlier stage disease. In particular, based on the success of early investigatorinitiated trials in which major pathologic response (MPR) rates of between $17 \%$ and $45 \%$ have been reported for 
ICB alone,${ }^{2-5}$ several large neoadjuvant trials are under way (Table 1). Despite the promise of neoadjuvant ICB, several challenges exist that merit discussion. It is critical that thoracic surgeons participate actively in the design and implementation of neoadjuvant trials, because the outcomes of these trials will depend heavily on patient selection and surgical expertise. Additionally, neoadjuvant trials present an excellent opportunity for surgeons to engage with and lead translational research. We present a brief overview of important considerations for surgeons regarding neoadjuvant ICB trials.

\section{WHICH PATIENTS SHOULD WE INCLUDE?}

Because the aim of the early phase I and II neoadjuvant trials has been mainly to establish safety and early efficacy, generally measured by MPR, inclusion criteria for enrollment have been rather broad. Trials have typically included patients older than 18 years with histologically or cytologically proven NSCLC of adenocarcinoma or squamous cell carcinoma histology. Although carcinoid tumors or small cell lung cancers have not been eligible, some trials allowed enrollment of carcinomas with neuroendocrine differentiation. Surgical resectability as judged by treating surgeons has been of paramount importance for inclusion. Therefore, most trials enrolled patients with resectable stages IB to IIIA NSCLC according to the American Joint Committee on Cancer 7th or 8th edition. Given the suboptimal overall survival of these patients, we support the concept of enrolling patients with stage II and III and even consider patients with stage IB disease. We strongly recommend that all patients undergo invasive mediastinal staging before enrollment on a trial with endobronchial ultrasound or mediastinoscopy. Performance status should be good (Eastern Cooperative Oncology Group 0-1), and patients must have adequate organ and bone marrow function.

Major exclusion criteria for ICB on current trials include common criteria such as history and treatment of lung cancer, pregnancy or lactation, other active malignancy within the last 2 years, and any other serious medical or psychologic illnesses. In particular, patients should also be

TABLE 1. Published manuscripts or abstracts describing early experiences with neoadjuvant immunotherapy followed by surgical resection for patients with non-small cell lung cancer

\begin{tabular}{|c|c|c|c|c|c|c|c|c|}
\hline Trial or series & Institution & $\begin{array}{c}\text { Neoadjuvant } \\
\text { treatment }\end{array}$ & $\begin{array}{c}\text { Published } \\
\text { vs abstract }\end{array}$ & $\begin{array}{c}\text { No. of } \\
\text { patients } \\
\text { enrolled/ } \\
\text { randomized }\end{array}$ & $\begin{array}{c}\text { Median } \\
\text { time to } \\
\text { surgery after } \\
\text { completion of } \\
\text { neoadjuvant } \\
\text { therapy }\end{array}$ & $\begin{array}{c}\text { No. of } \\
\text { patients } \\
\text { undergoing } \\
\text { resection } \\
(\%)\end{array}$ & $\begin{array}{l}\text { Grade } 3 \text { or } 4 \\
\text { complications }\end{array}$ & $\begin{array}{c}\text { MPR } \\
\text { (intention- } \\
\text { to-treat and } \\
\text { surgically } \\
\text { resected) }\end{array}$ \\
\hline NCT02259621 2,6 & MSK/JHMI & Nivolumab & Published & 21 & $18 \mathrm{~d}(11-29)$ & $20(95 \%)$ & $\begin{array}{l}50 \% \\
\quad(\text { any } \\
\text { morbidity) }\end{array}$ & $\begin{array}{c}9(43 \% \\
45 \%)\end{array}$ \\
\hline $\begin{array}{l}\text { NCT03158129 } \\
\quad\left(\text { NEOSTAR }^{3}\right)\end{array}$ & $\begin{array}{l}\text { MD } \\
\text { Anderson } \\
\text { Cancer } \\
\text { Center }\end{array}$ & $\begin{array}{l}\text { Nivolumab vs } \\
\text { Nivolumab }+ \\
\text { ipilimumab }\end{array}$ & $\begin{array}{l}\text { Abstract ASCO } \\
\text { 2019/WCLC } \\
2019\end{array}$ & 44 & $31 \mathrm{~d}(21-87)$ & $39(89 \%)$ & NR & $\begin{array}{c}11(25 \%, \\
30 \%) \\
19 \% \mathrm{~N} \text { vs } \\
44 \% \mathrm{~N}+\mathrm{I}\end{array}$ \\
\hline $\begin{array}{c}\text { NCT03081689 } \\
\left(\text { NADIM }^{7}\right)\end{array}$ & $\begin{array}{l}\text { Spanish } \\
\text { Lung } \\
\text { Cancer } \\
\text { Group }\end{array}$ & $\begin{array}{l}\text { Nivolumab }+ \\
\text { paclitaxel and } \\
\text { carboplatin }\end{array}$ & $\begin{array}{l}\text { Abstract ASCO } \\
2019\end{array}$ & 46 & $\begin{array}{l}\text { NR (target } \\
\text { 3-4 wk after } \\
\text { last dose) }\end{array}$ & $41(89 \%)$ & $17 \%$ & $\begin{array}{c}35(76 \%, \\
85 \%)\end{array}$ \\
\hline $\begin{array}{c}\text { NCT02927301 } \\
\left(\mathrm{LCMC}^{4}\right)\end{array}$ & Multicenter & Atezolizumab & $\begin{array}{l}\text { Abstract ASCO } \\
\text { 2019/AATS } \\
2019\end{array}$ & 101 & $\begin{array}{l}\text { NR }(17 \% \\
\text { with delays } \\
\text { to surgery) }\end{array}$ & $90(89 \%)$ & $6 \%$ & $\begin{array}{c}15(15 \%, \\
17 \%)\end{array}$ \\
\hline NCT02904954 & $\begin{array}{l}\text { Weill } \\
\text { Cornell }\end{array}$ & $\begin{array}{c}\text { Durvalumab vs } \\
\text { durvalumab }+ \\
8 \text { Gy } \times 3\end{array}$ & $\begin{array}{l}\text { Abstract } \\
\text { WCLC } \\
\text { 2019/AATS } \\
2019\end{array}$ & 34 & $\begin{array}{l}\text { NR (target 1-2 } \\
\text { wk after last } \\
\text { dose) }\end{array}$ & $30(88 \%)$ & $17 \%$ & $\begin{array}{c}11(32 \%, \\
37 \%) \\
6 \% \mathrm{D} \\
\text { vs } 53 \% \\
\mathrm{D}+\mathrm{SBRT}\end{array}$ \\
\hline $\begin{array}{r}\text { NCT01820754 } \\
\left(\text { TOP } 1201^{8}\right)\end{array}$ & Duke & $\begin{array}{c}\text { Chemotherapy }+ \\
\text { Ipilimumab }\end{array}$ & Published & 24 & NR & $13(54 \%)$ & NR by grade & $\begin{array}{c}2(8 \%, \\
15 \%) \\
\text { Only } \\
\text { reported } \\
\text { CPR }\end{array}$ \\
\hline
\end{tabular}

MPR, Major pathologic response; MSK/JHMI, Memorial Sloan Kettering/Johns Hopkins Medicine Institution; ASCO, American Society of Clinical Oncology; WCLC, World Conference on Lung Cancer; NR, not reported; AATS, American Association for Thoracic Surgery; SBRT, stereotactic body radiotherapy. 
questioned and excluded for immune-related disorders, including autoimmune diseases, pneumonitis, hepatitis B or $\mathrm{C}$ infection, human immunodeficiency virus or acquired immunodeficiency syndrome, and corticosteroid use (doses $>10 \mathrm{mg}$ per day) or prior treatment with any immunotherapy.

Considering the cost and selective efficacy of ICBs in the metastatic setting, there has been an intense search for predictive biomarkers of response. Many metastatic lung cancer trials stratified patients according to programmed death ligand 1 (PD-L1) expression on tumor cells or tumor mutation burden. ${ }^{9-11}$ Current neoadjuvant studies do not require percent PD-L1 expression or tumor mutation burden for enrollment; however, a myriad of biomarkers have been intensely studied in these trials. Questions remain whether patients with targetable mutations such as EGFR, ALK, ROS1, or BRAF should be enrolled on neoadjuvant immunotherapy trials. Although there is evidence in the metastatic setting that cancers with these mutations respond well to targeted therapies and poorly to immunotherapy, ${ }^{12,13}$ evidence in an early-stage or locally advanced setting is lacking. Despite the fact that there are other ongoing trials testing targeted therapies in the neoadjuvant setting, many ongoing immunotherapy neoadjuvant therapy trials also include these patients, mainly because mutation testing in early-stage disease is currently not considered standard of care and not routinely covered by insurance companies. However, 2 phase III randomized placebo controlled trials mandate testing for EGFR mutation. For example, the AEGEAN (NCT03800134) multicenter international phase III randomized trial of neoadjuvant chemotherapy plus durvalumab versus chemotherapy alone requires EGFR and ALK testing as well as tumor PD-L1 status; however, the trial will allow $20 \%$ of patients with EGFR mutation to be accrued. On the contrary, the phase III Checkmate 77T (NCT04025879) randomized, double-blind, placebocontrolled trial of neoadjuvant chemotherapy plus nivolumab versus chemotherapy alone requires EGFR and ALK targetable mutation testing and excludes these patients from enrollment; this trial enrolls those with clinical stage IIA to IIIB according to the American Joint Committee on Cancer 8th edition. Notably, in the LCMC3 and Cornell single-agent trials, no patients with EGFR mutations had an MPR in early reports. ${ }^{4,5}$ These tumors may simply be less antigenic because of lower mutation burden. Therefore, we recommend excluding them from similar single-agent trials. It remains to be seen whether patients with driver mutations may respond to ICB with combination chemotherapy in the neoadjuvant setting, but consideration should be made to at least limiting inclusion similar to the AEGEAN study. Further analyses from ongoing trials are needed to discover potential biomarkers that may be used in the future to stratify patients for neoadjuvant immunotherapy alone or concomitant chemotherapy and immunotherapy. The likely next step for improved patient selection for neoadjuvant strategies will be mandatory driver mutation testing.

\section{WHAT IS THE OPTIMAL DURATION AND OPTIMAL COMBINATION OF NEOADJUVANT THERAPY?}

In the neoadjuvant setting, a balance needs to be struck to allow patients enough time to respond to preoperative therapy, but not to overly delay loco-regional disease control and potentially curative surgical therapy in those patients who do not respond. We believe that the optimal duration depends on whether or not ICB is given as stand-alone therapy versus given in conjunction with cytotoxic therapy. For patients receiving mono or dual immunotherapy alone, published or presented trials have generally given only 2 to 3 cycles of neoadjuvant ICB..$^{2-5}$ With such a regimen, it is possible to get patients to the operating room within 4 to 6 weeks after starting therapy. Surgery soon after completion of therapy may have the advantage of avoiding or at least decreasing the intraoperative fibrosis that has been described after prolonged ICB or after long delays between completion of therapy and surgery. ${ }^{6}$ With neoadjuvant regimens that incorporate chemotherapy with or without radiation, preoperative treatment can be extended because potential nonresponders are at least receiving standard-of-care neoadjuvant cytotoxic treatment. The NADIM trial (NCT03081689) gave 3 cycles of neoadjuvant ICB and chemotherapy, and patients underwent operation 3 to 4 weeks after the final cycle. ${ }^{7}$ Recently completed or accruing trials, the CheckMate-816, Impower030, and KEYNOTE-671, all have similar structures.

The optimal combination of neoadjuvant therapy remains to be determined. Clearly, on the basis of MPR rates in early trials with ICB alone, many patients will respond as well or better than historical chemotherapy controls, which achieved MPR rates of approximately $15 \%$. However, in view of the unpublished results of the NADIM trial, which reported an unprecedented $83 \%$ MPR rate (which still needs to be validated), we anticipate that overall MPR rates will be higher with combination therapy. ${ }^{7}$ The results of other phase II trials of combination chemoimmunotherapy are eagerly anticipated. Whether the MPR rate will translate to a long-term survival advantage is not currently known, and the Food and Drug Administration does not yet recognize the MPR rate as a sufficiently powerful surrogate of survival to approve neoadjuvant drug regimens based on MPR. For now, we will need to wait for event-free, disease-free, or overall survival readout from these trials. Given the historically small survival benefit $(5 \%)$ of neoadjuvant and adjuvant chemotherapy for surgically resectable patients, we encourage continued patient enrollment on investigator-initiated and cooperative group trials with arms using ICB alone to better understand 
which patients may be able to completely avoid chemotherapy. ${ }^{14}$

\section{WHAT SIDE EFFECTS SHOULD BE ANTICIPATED DURING PREOPERATIVE MANAGEMENT?}

Overall, ICIs are well tolerated. The most common side effect noted in neoadjuvant trials to date has been fatigue. However, ICB disinhibition of T cells may result in offtarget effects due to immune activation and inflammation. Clinical sequelae of these effects are termed "immunerelated adverse events" and may manifest in various organ systems. The majority of data regarding immune-related adverse events come from medical oncology studies of patients with advanced disease. Data regarding immunerelated adverse events in the neoadjuvant setting are limited but will be augmented by forthcoming large prospective trials.

Pneumonitis is the most common pulmonary toxicity of ICB therapy and is the most relevant to patients receiving ICB therapy in the neoadjuvant setting because severe pneumonitis could potentially exclude patients from operative therapy. Large retrospective series and meta-analyses report an overall pneumonitis rate of approximately $5 \%$ with slightly higher rates in the setting of dual checkpoint inhibition versus monotherapy. ${ }^{15,16}$ However, single-center studies within these analyses have reported rates as high as $19 \%$. Time to onset of pneumonitis is variable, and clinical risk factors remain to be elucidated. Generally, pneumonitis tends to occur after multiple ICB doses; however, it can occur even after a single dose. Significant pneumonitis has been rare in neoadjuvant trials to date, but in some cases has caused significant hypoxia requiring high-dose steroids and a delay in surgery. Additional immune-related adverse events in patients treated with ICB for NSCLC include endocrinopathies (hypothyroidism, hypopituitarism, and adrenal insufficiency), gastrointestinal toxicities (colitis, hepatitis), and cardiovascular (myocarditis and pericarditis). The National Comprehensive Cancer Network and American Society of Clinical Oncology have issued guidelines for recognition and management of immune-related adverse events. ${ }^{17,18}$ The mainstay of treatment of immune-related adverse events is corticosteroids, with few patients requiring additional immunosuppression.

\section{HOW MANY PATIENTS WILL NOT MAKE IT TO SURGERY AND WHY?}

Despite the wide range of possible immune-related side effects, in neoadjuvant ICB studies to date most patients have completed neoadjuvant treatment and undergone resection. General "falloff" in ICB trials is approximately $10 \%$, which is similar to that with neoadjuvant chemotherapy alone. In the study by Forde and colleagues, ${ }^{2}$ in which patients received 2 doses of single-agent nivolumab,
20 of 21 patients underwent resection (1 patient was unresectable at exploration) without any delays to planned surgery. A single patient received only 1 dose of nivolumab and was taken to surgery early because of postobstructive pneumonia. The NEOSTAR investigators at the American Society of Clinical Oncology annual meeting in 2019 reported that 5 of 44 patients ( 1 in the single-agent nivolumab arm and 4 in nivolumab/ipilimumab arm) did not undergo resection, with grade 3 hypoxia in the patients with nivolumab only. ${ }^{3}$ In the LCMC3 study, 90 of 101 patients (89\%) (neoadjuvant atezolizumab) underwent planned resection. ${ }^{4}$ Most patients not undergoing resection demonstrated progressive disease $(n=5)$ or withdrew consent $(n=4)$. Likewise, in the Weill-Cornell neoadjuvant durvalumab/ stereotactic body radiotherapy study, the majority of patients (30/34) underwent planned resection. ${ }^{5}$ In the NADIM trial, 41 of 46 patients $(89 \%)$ underwent resection after chemo-immunotherapy.

Data from published studies and those presented in abstract form do not suggest undue delays to surgery or frequent treatment toxicities precluding surgical resection. As larger scale prospective studies report surgical outcomes, more data regarding this concern will become available.

\section{HOW SHOULD PATIENTS BE RESTAGED AND HOW SHOULD CLINICAL RESPONSE BE ASSESSED?}

At this time, all patients enrolled in neoadjuvant immunotherapy trials should have "resectable" disease as judged by a thoracic surgeon before trial enrollment. Hope or assumption that marginally resectable patients will be "downstaged" after 3 or 4 cycles of immunotherapy with or without chemotherapy and become "resectable" should be resisted until more data are available. Radiographic restaging is a component of all neoadjuvant immunotherapy trials and generally includes both computed tomography and combined positron emission tomography computed tomography. The aim of radiographic restaging is to measure therapeutic response within the primary tumor and lymph nodes and to ensure there is no disease progression, which would result in treatment plan change. Criteria for the response evaluation are according to the revised Response Evaluation Criteria in Solid Tumors guidelines version 1.1. ${ }^{19}$ Considering the fact that most checkpoint inhibitor drugs are infused every 2 or 3 weeks for just 2 or 3 doses, it is biologically unlikely that the primary cancer would significantly progress during the time of neoadjuvant therapy, even if there is no response to therapy. However, there have been reports of both pseudo-progression and hyper-progression on immunotherapy for lung cancer, mainly in the metastatic setting. ${ }^{20-22}$. Pseudo-progression is defined as an initial tumor enlargement followed by tumor regression and was 
observed in approximately $3 \%$ of patients with NSCLC treated with nivolumab monotherapy.

As one can imagine, pseudo-progression is difficult to report in the neoadjuvant setting because the majority of patients undergo resection soon after completing therapy; therefore, there is only a brief period to observe tumor regression/progression. However, it should be noted that patients with primary tumor pseudo-progression may experience better long-term outcomes. Hyperprogression is defined as a true rapid disease progression associated with patients' clinical deterioration. It has been reported with immunotherapy in lung and other cancers in $4 \%$ to $29 \%$ of metastatic patients. ${ }^{20,22}$ There is no clear consensus regarding the mechanism of this phenomenon, and some believe that it merely represents aggressive tumor biology, but it is possible that more patients may progress rapidly on immunotherapy than on chemotherapy. Disease progression on neoadjuvant immunotherapy has been reported. ${ }^{3,5}$ However; it is not clear that this represents hyperprogression due to ICB versus typical disease progression.

Although the primary outcomes of these trials focus on tumor responses, mainly pathologic but also radiographic, it is important to highlight a new phenomenon observed within lymph nodes: nodal immune flare (NIF). NIF represents an apparent but false de novo cancer progression within mediastinal or other lymph nodes after neoadjuvant immunotherapy. In the NEOSTAR trial after both nivolumab and nivolumab and ipilimumab, patients who demonstrated NIF had no pretreatment evidence of cancer on nodal staging before immunotherapy both by invasive biopsy and by the lack of ${ }^{18}$ FDG uptake within lymph nodes. ${ }^{3}$ After 3 cycles of immunotherapy, at least 5 (of 44) patients demonstrated increased nodal size and ${ }^{18}$ FDG uptake within mediastinal and other lymph nodes, suggesting nodal disease progression. However, the pathologic assessment of these lymph nodes demonstrated de novo nonnecrotizing granulomas not present before therapy but present uniformly in all resected lymph nodes. This observation suggests a systemic inflammatory reaction to immunotherapy in some patients. Further data on this phenomenon are forthcoming. The $10 \%$ rate of NIF in patients in the NEOSTAR trial highlights the importance of repeat invasive staging if there is evidence of new radiographic nodal disease progression to ensure that patients are not erroneously directed away from definitive surgical therapy.

WHAT IS THE BEST SURGICAL APPROACH AND RESECTION STRATEGY AFTER NEOADJUVANT IMMUNE CHECKPOINT BLOCKADE AND IS FIBROSIS/INFLAMMATION A CONCERN?

As with any postinduction lung resection, procedures after immunotherapy protocols require particular attention to perioperative planning. In regard to surgical approach, there were initial concerns that post-treatment changes may make minimally invasive resections challenging. Several series have now demonstrated that in many cases VATS or robotic resections are feasible. ${ }^{2-5}$ However, conversion to thoracotomy is not uncommon, and the rates of conversion vary in these early cohorts. For instance, the initial Memorial Sloan Kettering/Johns Hopkins Medicine Institution study of patients receiving preoperative nivolumab included 13 resections that were attempted with a VATS or robotic approach, and approximately half $(54 \%)$ were converted to thoracotomy. ${ }^{2}$ Likewise, in a study from Yang and colleagues ${ }^{8}$ at Duke, which used preoperative chemotherapy plus ipilimumab, 12 of 13 patients were planned for a minimally invasive approach and the conversion rate was $25 \%$. Of note, the study directly compared these outcomes with a historical cohort of 42 postchemotherapy cases from the authors' institution that demonstrated a conversion rate of $39 \%$.

It remains to be determined what variables affect operative difficulty. Although it has been suggested that factors such as hilar nodal involvement or degree of treatment effect may play a role, these will need to be evaluated in larger studies. Along these lines, comparisons between standard chemotherapy induction protocols and immunotherapy protocols have renewed interest in characterizing and quantifying post-treatment changes such as peri-hilar inflammation or fibrosis. Although these assessments remain subjective, initial efforts have been made to standardized descriptors. A recent presentation of the Weill Cornell series proposed a basic scoring system for hilar fibrosis. Although the proposed system is fairly simple (grade $0=$ no fibrosis, grade $1=$ moderate fibrosis that prolongs the procedure, grade $2=$ dense fibrosis that changes the operative approach), it may serve as a framework for future efforts. Such a system, particularly one that can relate preoperative data to degree of fibrosis, would be an important tool for preoperative planning.

The safety of pneumonectomy after induction immunotherapy is equally unclear. Published abstracts from the NEOSTAR trial of induction nivolumab or nivolumab plus ipilimumab identified 2 bronchopleural fistulas (BPFs) $(6 \%)$ and 8 prolonged air leaks $(24 \%)$ in 34 operative patients. ${ }^{3}$ A recent update of the data from the Cornell group showed a similar rate of BPF $(2 / 32,6 \%)$ in patients resected after neoadjuvant durvalumab and stereotactic radiation. ${ }^{5}$ The study from Duke shows a prolonged air leak rate of $15 \% .{ }^{8}$ This raises concerns regarding bronchial or parenchymal healing after these treatment modalities, which may have implications for patients undergoing pneumonectomy. The number of patients undergoing pneumonectomy in early trials is small (Memorial Sloan Kettering/Johns Hopkins Medicine Institution $=2$, Duke $=1$ ). 
Therefore, further information is needed before true measures of safety can be assessed.

\section{SHOULD WE EXPECT HIGHER RATES OF POSTOPERATIVE COMPLICATIONS IN THESE PATIENTS?}

On a broader level, overall morbidity and mortality after induction immunotherapy trials appear to be grossly similar to comparative cohorts receiving preoperative chemotherapy or chemoradiation. Both the published series report no 30-day mortality in 33 patients. ${ }^{2,8}$ The NEOSTAR study included 1 death ( $3 \%$ mortality) due to BPF in a patient treated with nivolumab preoperatively. ${ }^{3}$ Aside from the air leak and BPF complications described, general morbidity was comparable to what would be expected in the setting of anatomic lung resection. The most common complications in these series include atrial fibrillation, urinary tract infection, and pneumonia. ${ }^{2,8}$ Most of these complications appear to be minor in severity. The median length of stay also appears consistently at approximately 4 to 5 days.

\section{HOW SHOULD MAJOR PATHOLOGIC RESPONSE BE DEFINED AND WHAT IS THE EXPECTED RATE?}

Previous trials, including the only one to be fully published, described MPR as no more than $10 \%$ viable tumor cells on routine hematoxylin-eosin staining. ${ }^{2}$ Because such an assessment can be challenging and open to interpretation, we recommend centralized evaluation by experienced pathologists, particularly for larger multi-institutional trials. We suggest not using a yes/no scale, but rather characterization of the absolute percentage of viable tumor cells on a gradient, ideally assessed by at least 2 pathologists. The absolute percentage likely has important implications. Additionally, it has been suggested that the clinical importance of the percentage of viable tumor cells may be dependent on different cutoff points for patients with adenocarcinoma and squamous cell carcinoma after chemotherapy, with patients with adenocarcinoma showing improved survival at lower rates of pathologic response. ${ }^{23}$ The relationship of overall survival to MPR after ICB still needs to be better characterized.

Nevertheless, MPR is a compelling short-term end point. MPR reported in the initial trial with nivolumab was a remarkable $45 \%$, with a $10 \%$ rate of complete pathologic response. ${ }^{2}$ It remains to be seen whether such a high MPR rate can be achieved in larger studies with single-agent ICB. In the comparable NEOSTAR arm with nivolumab alone, MPR was $17 \%$ with a $9 \% \mathrm{CPR}^{3}$ MPR rates with neoadjuvant atezolizumab alone were $19 \%$ in the LCMC3 study. ${ }^{4}$ Therefore, it seems that rates of $15 \%$ to $20 \%$ can generally be expected with single-agent immunotherapy. On the other hand, combination therapy has been shown to generate higher MPR rates. In the NEOSTAR trial, nivolumab with ipilimumab had a $33 \%$ rate of MPR, including
$29 \%$ of patients who had CPR. ${ }^{3}$ In the Weill Cornell trial, MPR was $6 \%$ with durvalumab alone versus $53 \%$ with nonablative radiation plus durvalumab. ${ }^{5}$ Finally, the NADIM trial has reported a remarkable $83 \%$ rate of MPR and $71 \%$ rate of CPR with nivolumab plus chemotherapy. ${ }^{7}$ Most of these data remain unpublished, and trials are still ongoing. It remains to be seen whether such high rates will be achieved with the larger enrolling trials. If so, such MPR rates would be markedly higher than rates reported with neoadjuvant chemotherapy $(\sim 15 \%)$ or even neoadjuvant chemoradiation and would suggest that a long-term survival advantage should be expected. Such results would likely push neoadjuvant ICB toward the standard of care for selected patients.

\section{CONCLUSIONS}

Multiple neoadjuvant ICB trials are under way or under development. These trials use a variety of ICB agents, as well as combination strategies. Neoadjuvant ICB may eventually become the standard of care for selected patients with locally and regionally advanced NSCLC based on improved efficacy over neoadjuvant chemotherapy. Nevertheless, several points must be kept in mind as we embark upon this new paradigm:

1. Patient selection and surgeon involvement in trial enrollment are critical.

2. Unique side effects may occur during neoadjuvant ICB but are generally manageable and do not exclude patients from surgery.

3. Progression during neoadjuvant ICB is rare, and radiographic assessment of response can be imprecise. Clinical suspicion of progression should be confirmed pathologically.

4. Minimally invasive operations are feasible, but these operations may be technically challenging and conversion should be considered if thoracoscopy does not provide for safe dissection.

5. Pneumonectomy should be performed cautiously until additional safety data are available.

6. Efforts should be made toward a more formal characterization and standardization of post-treatment tissue changes that affect surgical resection.

7. All future trials should include detailed assessment of treatment-related and nontreatment-related complications.

8. Pathologic assessment of MPR should be further standardized and closely correlated with long-term recurrence and survival to become a reliable surrogate of therapeutic efficacy in neoadjuvant trials.

\section{Conflict of Interest Statement}

Dr Stiles receives consulting/speaker fees from AstraZeneca, Bristol-Myers Squibb, Pfizer, Genentech, Flame 
Biosciences, Ribon Therapeutics, Gala Therapeutics, Medtronic, and WebMD, and is a board member of Lung Cancer Research Foundation. Dr Sepesi receives consulting fees from Bristol-Myers Squibb. Dr Broderick is a consultant for Bristol Myers Squibb. Dr Bott previously received consulting fees from AstraZeneca.

The Journal policy requires editors and reviewers to disclose conflicts of interest and to decline handling or reviewing manuscripts for which they may have a conflict of interest. The editors and reviewers of this article have no conflicts of interest.

\section{References}

1. Siegel RL, Miller KD, Jemal A. Cancer statistics, 2020. CA Cancer J Clin. 2020; 70:7-30.

2. Forde PM, Chaft JE, Smith KN, Anagnostou V, Cottrell TR, Hellman MD, et al. Neoadjuvant PD-1 blockade in resectable lung cancer. N Engl J Med. 2018;378: 1976-86.

3. Cascone T, William WN, Weissferdt A, Lin HY, Leung CH, Carter BW, et al. Neoadjuvant nivolumab or nivolumab plus ipilimumab for resectable nonsmall cell lung cancer (NSCLC): clinical and correlative results from the NEOSTAR study. J Clin Oncol. 2019;37(15_suppl):8504 (presented at ASCO 2019).

4. Kwiatkowski DJ, Rusch VW, Chaft JE, Johnson BE, Nicholas A, Wistuba II, et al. Neoadjuvant atezolizumab in resectable non-small cell lung cancer (NSCLC): interim analysis and biomarker data from a multicenter study (LCMC3). J Clin Oncol. 2019;37(15_suppl):8503 (presented at ASCO 2019).

5. Altorki N, Borczuk A, Saxena A, Port J, Stiles B, Lee B, et al. Neoadjuvant durvalumab with or without subablative stereotactic radiotherapy in patients with resectable NSCLC (NCT02904954). J Thorac Oncol. 2019;14:S746.

6. Bott MJ, Yang SC, Park BJ, Adusumilli PS, Rusch VW, Isbell JM, et al. Initial results of pulmonary resection after neoadjuvant nivolumab in patients with resectable non-small cell lung cancer. J Thorac Cardiovasc Surg. 2019;158:269-76.

7. Provencio M, Nadal E, Insa A, Garcia-Campelo R, Rubio JC, Domine M, et al. Neoadjuvant chemo-immunotherapy for the treatment of stage IIIA resectable non-small-cell lung cancer (NSCLC): a phase II multicenter exploratory study - final data of patients who underwent surgical assessment. J Clin Oncol. 2019;37(15_suppl):8509 (presented at ASCO 2019).

8. Yang CJ, McSherry F, Mayne NR, Wang X, Berry MF, Tong B, et al. Surgical outcomes after neoadjuvant chemotherapy and ipilimumab for non-small cell lung cancer. Ann Thorac Surg. 2018;105:924-9.

9. Gandhi L, Rodríguez-Abreu D, Gadgeel S, Esteban E, Felip E, De Angelis F, et al. Pembrolizumab plus chemotherapy in metastatic non-small cell lung cancer. N Engl J Med. 2018;378:2078-92.

10. Mok TSK, Wu YL, Kudaba I, Kowalski DM, Cho BC, Turna HZ, et al. Pembrolizumab versus chemotherapy for previously untreated, PD-L1-expressing, locally advanced or metastatic non-small-cell lung cancer (KEYNOTE-042): a randomised, open-label, controlled, phase 3 trial. Lancet. 2019;393:1819-30.

11. Hellmann MD, Ciuleanu TE, Pluzanski A, Lee JS, Otterson GA, AudigierValette C, et al. Nivolumab plus ipilimumab in lung cancer with a high tumor mutational burden. N Engl J Med. 2018;378:2093-104.

12. Lisberg A, Cummings A, Goldman JW, Bornazyan K, Reese N, Wang T, et al. A Phase II study of pembrolizumab in EGFR-mutant, PD-L1+, tyrosine kinase inhibitor naïve patients with advanced NSCLC. J Thorac Oncol. 2018; $13: 1138-45$.

13. Mhanna L, Guibert N, Milia J, Mazieres J. When to consider immune checkpoint inhibitors in oncogene-driven non-small cell lung cancer? Curr Treat Options Oncol. 2019;20:60.

14. Blumenthal GM, Bunn PA Jr, Chaft JE, McCoach CE, Perez EA, Scagliotti GV, et al. Current status and future perspectives on neoadjuvant therapy in lung cancer. J Thorac Oncol. 2018;13:1818-31.

15. Naidoo J, Wang X, Woo KM, Iyriboz T, Halpenny D, Cunningham J, et al. Pneumonitis in patients treated with anti-programmed death-1/programmed death ligand 1 therapy. J Clin Oncol. 2017;35:709-17.

16. Nishino M, Giobbie-Hurder A, Hatabu H, Ramaiya NH, Hodi FS. Incidence of programmed cell death 1 inhibitor-related pneumonitis in patients with advanced cancer: a systematic review and meta-analysis. JAMA Oncol. 2016;2:1607-16.

17. Thompson JA. New NCCN guidelines: recognition and management of immunotherapy-related toxicity. J Natl Compr Canc Netw. 2018;16(Suppl 5): 594-6.

18. Brahmer JR, Lacchetti C, Schneider BJ, Atkins MB, Brassil KJ, Caterino JM, et al. Management of immune-related adverse events in patients treated with immune checkpoint inhibitor therapy: American Society of Clinical Oncology clinical practice guideline. J Clin Oncol. 2018;36:1714-68.

19. Eisenhauer EA, Therasse P, Bogaerts J, Schwartz LH, Sargent D, Ford R, et al. New response evaluation criteria in solid tumours: revised RECIST guideline (version 1.1). Eur J Cancer. 2009;45:228-47.

20. Fujimoto D, Yoshioka H, Kataoka Y, Morimoto T, Hata T, Kim YH, et al. Pseudoprogression in previously treated patients with non-small cell lung cancer who received nivolumab monotherapy. J Thorac Oncol. 2019;14:468-74.

21. Frelaut M, Le Toruneau C, Borcoman E. Hyperprogression under immunotherapy. Int J Mol Sci. 2019;20:2674.

22. Ferrara R, Mezquita L, Texier M, Lahmar J, Audigier-Valette C, Tessonnier L, et al. Hyperprogressive disease in patients with advanced non-small cell lung cancer treated with PD-1/PD-L1 inhibitors or with single-agent chemotherapy. JAMA Oncol. 2018;4:1543-52.

23. Qu Y, Emoto K, Eguchi T, Aly RG, Zheng H, Chaft JE, et al. Pathologic assessment after neoadjuvant chemotherapy for NSCLC: importance and implications of distinguishing adenocarcinoma from squamous cell carcinoma. J Thorac Oncol. 2019;14:482-93.

Key Words: immunotherapy, neoadjuvant, non-small cell lung cancer, surgery 